\title{
Treatment of Hepatocellular Carcinoma with Portal Vein Tumor Thrombosis
}

\author{
Junyi Shi', Ai Shen',3, Tong Mou ${ }^{3}$, Zhongjun $\mathrm{Wu}^{3^{*}}$ \\ ${ }^{1}$ Department of Oncology, The First Affiliated Hospital of Chongqing Medical University, Chongqing, China \\ ${ }^{2}$ Hepatobiliary Pancreatic Tumor Center, Chongqing University Cancer Hospital, Chongqing, China \\ ${ }^{3}$ Department of Hepatobiliary Surgery, The First Affiliated Hospital of Chongqing Medical University, Chongqing, China \\ Email: judyshijunyi@163.com, shenai200808@163.com, mutong_9@hotmail.com, „wzjtcy@126.com
}

How to cite this paper: Shi, J.Y., Shen, A., Mou, T. and Wu, Z.J. (2020) Treatment of Hepatocellular Carcinoma with Portal Vein Tumor Thrombosis. Journal of Cancer Therapy, 11, 115-123.

https://doi.org/10.4236/jct.2020.113010

Received: February 20, 2020

Accepted: March 3, 2020

Published: March 6, 2020

Copyright $\odot 2020$ by author(s) and Scientific Research Publishing Inc. This work is licensed under the Creative Commons Attribution International License (CC BY 4.0).

http://creativecommons.org/licenses/by/4.0/

\section{(c) (i) Open Access}

\begin{abstract}
The prognosis of hepatocellular carcinoma (HCC) with portal vein tumor thrombosis (PVTT) is very poor although sorafenib is recommended as the first-line treatment. Therefore, an effective treatment regime is needed for treating HCC with PVTT. This review summarized seven potential treatment regimes which including transarterial chemoembolization (TACE), TACE combined with sorafenib, TACE combined with radiotherapy (RT), hepatectomy, hepatic arterial infusion chemotherapy (HAIC), HAIC combined with sorafenib and HAIC combined with RT in the treatment of HCC with PVTT. In conclusion, hepatectomy or the combination of HAIC and sorafenib may be a more effective modality in the treatment of HCC patients with type I - II PVTT. HAIC combined with or without sorafenib/RT or the combination of RT and TACE is an alternative treatment choice for HCC patients with type III - IV PVTT. Further randomized controlled studies are warranted.
\end{abstract}

\section{Keywords}

Hepatocellular Carcinoma, Portal Vein Tumor Thrombosis, Hepatic Arterial Infusion Chemotherapy

\section{Introduction}

Hepatocellular carcinoma (HCC) is one of the seven leading cancer in the world and the third leading cause of cancer related death [1]. HCC is asymptomatic during the early stage, most HCC are diagnosed in intermediate or advanced stage so that it is not candidate for surgery and thus affects the prognosis of patients. According to the American Association for the Study of Liver Diseases 
(AASLD) guidelines, only $10 \%$ of HCC patients are suitable for surgical treatment [2]. In terms of AASLD, European Association for the Study of the liver and Asian Pacific Association for the Study of the Liver guidelines, transarterial chemoembolization (TACE) is the first choice of intermediate stage HCC patients and sorafenib is the first-line treatment of advanced HCC [2] [3] [4]. However, approximately $35 \%-45 \%$ of advanced HCC accompanied by portal vein tumor thrombosis (PVTT) [2] [5] [6] which can cause several severe prognosis-related complications such as portal hypertension, upper gastrointestinal hemorrhage and hepatic encephalopathy. A systematic review has shown that the median overall survival (mOS) of advanced HCC patients with PVTT is only about 2.7 months, significantly lower than that of patients without PVTT in which mOS is 24.4 months [7]. According to current guidelines, sorafenib is still recommended as the first-line treatment for HCC with PVTT [2], and this recommendation is based on two phase III clinical trials (SHARP and ORIENTAL) [5] [8]. However, Although the two trials showed a statistical significantly difference in survival between sorafenib and placebo (Harzard ratio(HR): 0.69, 90\% Confidence interval (CI): $0.53-0.89$ ), the mOS of patients in sorafenib group was only 47 days longer than that in placebo group (184 vs. 137 days) [9]. So, the efficacy of sorafenib in the treatment of HCC patients with PVTT is limited. Other more effective treatment regimes are warranted. Therefore, this review will summarize and discuss the potential treatment modality in the treatment of HCC with PVTT. This view was conducted based on an online search on Pubmed by using the search items "hepatocelluar carcinoma" and "portal vein tumor thrombosis" combined with "sorafenib" or "transaterial chemoembolization/trasaterial embolization" or "radiotherapy" or "hepatectomy/hepatic resection" or "hepatic aterial infusion chemotherapy" with language limited to English, before February 5, 2020.

\section{TACE}

Although TACE is recommended as the first-line treatment of intermediate HCC [2] [3] [4], it is also effective for advanced HCC with PVTT. A prospective study conducted by Luo et al. included 164 patients showed that the mOS of HCC patients with PVTT in TACE treatment group was 7.1 months, significantly longer than that in the conservative treatment group which was 4.1 months, especially for patients with type I - II PVTT which mOS was 10.2 and 5.2 months in TACE group and conservative group, respectively. Additionally, the benefit of TACE for type III - IV PVTT was also better than that of conservative treatment (mOS: 5.3 vs 3.4 months) [10]. Niu's study demonstrated that the mOS of HCC patients with PVTT treated with TACE was 8.67 months, much longer than that of conservative treatment which was only 1.4 months [11]. A multicenter study showed that the mOS of patients in TACE group was 9 months and 6 months, respectively, compared with that in conservative treatment group. For type I, II, III and IV PVTT, the mOS of TACE and conservative 
treatment group was 19, 12, 9, 6 and 12, 7, 7, 5 months, respectively. It showed that TACE was significantly better than conservative group for treating type I, II and III PVTT, but the benefit of TACE in type IV PVTT is not significant [12]. In addition, Pinter's study shows that TACE is as effective as sorafenib in the treatment of HCC with PVTT, and can be used as an alternative to sorafenib [13]. However, TACE is only a locoregional therapy and it may lead to severe liver dysfunction for type III - IV PVTT [14], so the usefulness of TACE in type III - IV PVTT is controversial.

\section{TACE Combined with Sorafenib}

Due to that TACE is a locoregional therapy, so combined with a systematic agent may improve the treatment efficacy. Choi's study showed that TACE combined with sorafenib was superior to sorafenib with respect to OS (HR: 0.64 and $0.48,95 \% \mathrm{CI}: 0.44,0.92, \mathrm{P}=0.02)$, but, there was a significant difference in the proportion of type III - IV PVTT patients between the two groups (24.5\% vs. 36.2\%) [15]. Although Yuan's study showed that TACE combined with sorafenib was superior to TACE alone in the treatment of hepatitis B-related HCC with PVTT (mOS was 13.0 and 7.0 months, respectively), the study did not report the complications after treatment [16]. In addition, a phase III randomized controlled trial in Japan showed that TACE combined with sorafenib had no significant advantage over TACE alone in the treatment of unresectable advanced HCC [17]. Also, TACE treatment may lead to severe liver dysfunction in the treatment of type III - IV PVTT [14], so the combination treatment should be further evaluated.

\section{TACE Combined with Radiotherapy}

Because the poor prognosis of patients with PVTT is mainly due to intrahepatic dissemination or complications resulting from portal hypertension, rather than the progression of the tumor itself, some researchers focused on treating PVTT to improve the prognosis of HCC. Radiotherapy (RT) was used for treating solid tumors for years, and it combined with TACE showed advantages in the treatment of HCC with PVTT. The combination of the two therapeutic methods has the following advantages: TACE can reduce the volume of tumor target so that increase the radiation dose of RT to PVTT and reduce the damage of normal liver tissue. RT can inhibit or kill the residual tumor after TACE. DSA can found the tumor lesions that CT or MRI cannot found, which is convenient for RT to sketching the target area [18]. A retrospective study of Lu showed that TACE combined with 3D-RT significantly prolonged the survival time of HCC patients with PVTT compared with TACE alone (mOS: 13.0 vs. 9.0 months, respectively) [19]. Li's propensity matching score study showed that TACE combined with 3D-RT was superior to TACE in the treatment of type II and III PVTT, with mOS of 12.5, 8.9 and 4.4, 4.0 months, respectively. However, there was no significant difference between the two treatment regime when treating HCC with 
type I and IV PVTT, with mOS of 23.7, 4.8 and 22.8, and 3.1 months, respectively [18]. Wang's study also showed that TACE combined with 3D-RT was significantly better than hepatectomy, TACE, TACE combined with sorafenib for type III PVTT, with mOS of 8.9, 6.0, 4.9 and 7.0 months, respectivey [20]. In addition, stereotactic body radiotherapy (SBRT) is regarded superior to 3D-RT due to its advantages of high accuracy, high dosage, high conformability and less treatment times. A study showed that the combination of SBRT and TACE can have some survival benefits compared with SBRT alone, but the combination may also damage the liver function [21]. Moreover, these studies are all retrospective studies, and the sample size is small. Prospective studies are still needed to verify the efficacy of TACE combined RT.

\section{Hepatectomy}

According to the guidelines, patients with HCC beyond Milan criteria are not suitable for hepatectomy. However, studies have shown that if the tumor can be completely removed, the patients can benefit from hepatectomy although HCC beyond Milan criteria [22] [23]. If the tumor and PVTT can be completely removed, patients can also obtain longer survival time and higher quality of life [7]. Peng's study showed that the 1-, 3-, 5-year survival rate of PVTT patients in hepatectomy and TACE group were $42.0 \%, 14.1 \%, 11.1 \%$ and $37.8 \%, 7.3 \%, 0.5 \%$, respectively. Subgroup analysis showed that for type I $(\mathrm{P}<0.001)$ and type II $(\mathrm{P}=0.002) \mathrm{PVTT}$, single tumor $(\mathrm{P}<0.001)$ and tumor diameter $>5 \mathrm{~cm}(\mathrm{P}<$ $0.001)$, the result favors hepatectomy [24]. Zheng's study showed that the 1 -, 3 -, and 5-year survival rates of PVTT patients in the hepatectomy group were $86.5 \%, 60.4 \%$, and $33.3 \%$, respectively, while those in the TACE group were $77.6 \%, 47.8 \%$, and $20.9 \%$, respectively $(\mathrm{P}=0.021)$. Univariate and multivariate analysis showed that the efficacy of hepatectomy was superior to that of TACE [25]. Lee's study showed that for HCC patients with PVTT, the survival time of patients in hepatectomy group was significantly longer than that in TACE or sorafenib group (mOS: 19.9, 6.6 and 6.2 months, respectively, $\mathrm{P}<0.001$ ) [26]. A large case series study showed that hepatectomy (mOS: 15.9, and 12.5 months, respectively) was significantly prong the survival than TACE (mOS: 9.3 and 4.9 months, respectively), TACE combined with sorafenib (mOS: 12.0 and 8.9 months, respectively), TACE combined with RT (12.2 and 10.6 months, respectively) in the treatment of HCC patients with type I and II PVTT [20]. These studies showed hepatectomy is beneficial in the treatment of HCC with PVTT, especially in type I - II PVTT.

\section{Hepatic Arterial Infusion Chemotherapy}

Hepatic arterial infusion chemotherapy (HAIC) is considered as an effective treatment for PVTT, which is widely used in Japan and Korea [27] [28] [29]. HAIC can increase and maintain the concentration of chemotherapeutic drugs in tumor cells. Theoretically, HAIC can treat HCC and PVTT. Compared with 
systemic chemotherapy, the incidence of adverse events can be significantly reduced due to the decrease of systemic distribution of chemotherapeutic drugs. Some retrospective studies showed that HAIC can improve the survival of HCC patients with PVTT, which is significantly better than sorafenib [28] [29]. The study of Nanako showed that continuous infusion of lipiodol and chemotherapeutics via hepatic artery could significantly prolong the survival period of HCC patients with type II - IV PVTT than sorafenib, and the mOS was 30.4 and 13.2 months, respectively [29]. A small randomized controlled trial involving only 58 patients showed that HAIC significantly prolong the survival time of HCC patients with type III - IV PVTT (mOS: 14.9 months vs 7.2 months) compared with sorafenib [27]. These studies showed the advantages of HAIC in the treatment of HCC with PVTT, but still need large scale clinical trials to verify because most of these studies were retrospective or small sample size studies.

\section{HAIC Combined with Sorafenib}

Because HAIC is a locoregional treatment, and advanced HCC may have potential metastasis, a systematic treatment drug is needed for comprehensive treatment. HAIC combined with sorafenib was used in many studies, and the result favors the combination regime. Nagai's study included 38 HCC patients with type III - IV PVTT. The mOS of the patients treated with HAIC and sorafenib was 315 days, which was significantly longer than that treated with HAIC alone (197days) [30]. A prospective randomized controlled trial recruited 247 HCC patients with PVTT showed that HAIC combined with sorafenib could significantly prolong the survival time (mOS: 13.37 vs. 7.13 months, respectively), and the progression-free survival time (median: 7.03 vs 2.6 months, respectively) of patients compared with sorafenib alone [31]. The mOS of patients in combination therapy group and sorafenib monotherapy group was 18.17 and 10.87 months, respectively in type I - II PVTT subgroup, 13.47 and 6.27 months respectively in type III PVTT subgroup, 9.47 and 5.5 months respectively in type IV PVTT subgroup [31]. These studies indicate the effectiveness and feasibility of HAIC combined with sorafenib in the treatment of HCC with PVTT.

\section{HAIC Combined with RT}

The purpose of HAIC combined with RT is to inhibit the growth of PVTT by $\mathrm{RT}$, protect the portal vein blood flow and prevent the deterioration of residual liver function, so as to achieve the maximum therapeutic effect of HAIC. Onishi's retrospective study showed that HAIC combined with RT can significantly improve the objective response rate of liver tumor $(52 \%$ vs. $18 \%, \mathrm{P}<0.01)$ and PVTT ( $45 \%$ vs. $18 \%, \mathrm{P}=0.01$ ) compared with HAIC monotherapy in the treatment of HCC patients with type III - IV PVTT, and its survival period is significantly prolonged (mOS: 12.4 vs. 5.7 months) [32]. Kodama's retrospective study showed that HAIC combined with RT is superior to sorafenib in the treatment of HCC with type III - IV PVTT with respect to PFS (median: 3.9 vs. 2.1 months) 
and OS (median: 9.9 vs. 5.3 months) [33]. However, these conclusions need to be verified by further well-designed clinical studies.

\section{Conclusion}

Current studies showed that hepatectomy or the combination of HAIC and sorafenib may be a more effective modality in the treatment of HCC patients with type I - II PVTT. HAIC combined with or without sorafenib/RT or, the combination of RT and TACE are potential beneficial regimens for the treatment of HCC with type III - IV PVTT. Further studies are warranted to verify these conclusions.

\section{Acknowledgements}

Junyi Shi and Ai Shen contributed equally to this review.

\section{Funding}

This review was funding from the graduate tutor team construction project of Chongqing Municipal Education Commission Foundation, China (No. dstd 201801)

\section{Conflicts of Interest}

The authors declare no conflicts of interest regarding the publication of this paper.

\section{References}

[1] Bray, F., Ferlay, J., Soerjomataram, I., Siegel, R.L., Torre, L.A. and Jemal, A. (2018) Global Cancer Statistics 2018: GLOBOCAN Estimates of Incidence and Mortality Worldwide for 36 Cancers in 185 Countries. CA: A Cancer Journal for Clinicians, 68, 394-424. https://doi.org/10.3322/caac.21492

[2] Heimbach, J.K., Kulik, L.M., Finn, R.S., Sirlin, C.B., Abecassis, M.M., Roberts, L.R., et al. (2018) AASLD Guidelines for the Treatment of Hepatocellular Carcinoma. Hepatology, 67, 358-380. https://doi.org/10.1002/hep.29086

[3] European Association for the Study of the Liver. Electronic Address EEE, European Association for the Study of the L (2018) EASL Clinical Practice Guidelines: Management of Hepatocellular Carcinoma. Journal of Hepatology, 69, 182-236.

[4] Omata, M., Cheng, A.L., Kokudo, N., Kudo, M., Lee, J.M., Jia, J., et al. (2017) AsiaPacific Clinical Practice Guidelines on the Management of Hepatocellular Carcinoma: A 2017 Update. Hepatology International, 11, 317-370. https://doi.org/10.1007/s12072-017-9799-9

[5] Llovet, J.M., Ricci, S., Mazzaferro, V., Hilgard, P., Gane, E., Blanc, J.F., et al. (2008) Sorafenib in Advanced Hepatocellular Carcinoma. The New England Journal of Medicine, 359, 378-390. https://doi.org/10.1056/NEJMoa0708857

[6] Llovet, J.M., Bustamante, J., Castells, A., Vilana, R., Ayuso Mdel, C., Sala, M., et al. (1999) Natural History of Untreated Nonsurgical Hepatocellular Carcinoma: Rationale for the Design and Evaluation of Therapeutic Trials. Hepatology, 29, 62-67. https://doi.org/10.1002/hep.510290145 
[7] Zhang, Z.Y., Dong, K.S., Zhang, E.L., Zhang, L.W., Chen, X.P. and Dong, HH. (2019) Resection Might Be a Meaningful Choice for Hepatocellular Carcinoma with Portal Vein Thrombosis: A Systematic Review and Meta-Analysis. Medicine, 98, e18362. https://doi.org/10.1097/MD.0000000000018362

[8] Cheng, A.L., Kang, Y.K., Chen, Z., Tsao, C.J., Qin, S., Kim, J.S., et al. (2009) Efficacy and Safety of Sorafenib in Patients in the Asia-Pacific Region with Advanced Hepatocellular Carcinoma: A Phase III Randomised, Double-Blind, Placebo-Controlled Trial. The Lancet Oncology, 10, 25-34. https://doi.org/10.1016/S1470-2045(08)70285-7

[9] Bruix, J., Cheng, A.L., Meinhardt, G., Nakajima, K., De Sanctis, Y. and Llovet, J. (2017) Prognostic Factors and Predictors of Sorafenib Benefit in Patients with Hepatocellular Carcinoma: Analysis of Two Phase III Studies. Journal of Hepatology, 67, 999-1008. https://doi.org/10.1016/j.jhep.2017.06.026

[10] Luo, J., Guo, R.P., Lai, E.C., Zhang, Y.J., Lau, W.Y., Chen, M.S., et al. (2011) Transarterial Chemoembolization for Unresectable Hepatocellular Carcinoma with Portal Vein Tumor Thrombosis: A Prospective Comparative Study. Annals of Surgical Oncology, 18, 413-420. https://doi.org/10.1245/s10434-010-1321-8

[11] Niu, Z.J., Ma, Y.L., Kang, P., Ou, S.Q., Meng, Z.B., Li, Z.K., et al. (2012) Transarterial Chemoembolization Compared with Conservative Treatment for Advanced Hepatocellular Carcinoma with Portal Vein Tumor Thrombus: Using a New Classification. Medical Oncology, 29, 2992-2997.

https://doi.org/10.1007/s12032-011-0145-0

[12] Xiang, X., Lau, W.Y., Wu, Z.Y., Zhao, C., Ma, Y.L., Xiang, B.D., et al. (2019) Transarterial Chemoembolization versus Best Supportive Care for Patients with Hepatocellular Carcinoma with Portal Vein Tumor Thrombusa Multicenter Study. European Journal of Surgical Oncology, 45, 1460-1467. https://doi.org/10.1016/j.ejso.2019.03.042

[13] Pinter, M., Hucke, F., Graziadei, I., Vogel, W., Maieron, A., Konigsberg, R., et al. (2012) Advanced-Stage Hepatocellular Carcinoma: Transarterial Chemoembolization versus Sorafenib. Radiology, 263, 590-599.

https://doi.org/10.1148/radiol.12111550

[14] Chung, J.W., Park, J.H., Han, J.K., Choi, B.I., Han, M.C., Lee, H.S., et al. (1996) Hepatic Tumors: Predisposing Factors for Complications of Transcatheter Oily Chemoembolization. Radiology, 198, 33-40.

https://doi.org/10.1148/radiology.198.1.8539401

[15] Choi, G.H., Shim, J.H., Kim, M.J., Ryu, M.H., Ryoo, B.Y., Kang, Y.K., et al. (2013) Sorafenib Alone versus Sorafenib Combined with Transarterial Chemoembolization for Advanced-Stage Hepatocellular Carcinoma: Results of Propensity Score Analyses. Radiology, 269, 603-611. https://doi.org/10.1148/radiol.13130150

[16] Yuan, J., Yin, X., Tang, B., Ma, H., Zhang, L., Li, L., et al. (2019) Transarterial Chemoembolization (TACE) Combined with Sorafenib in Treatment of HBV Background Hepatocellular Carcinoma with Portal Vein Tumor Thrombus: A Propensity Score Matching Study. BioMed Research International, 2019, Article ID: 2141859. https://doi.org/10.1155/2019/2141859

[17] Kudo, M., Imanaka, K., Chida, N., Nakachi, K., Tak, W.Y., Takayama, T., et al. (2011) Phase III Study of Sorafenib after Transarterial Chemoembolisation in Japanese and Korean Patients with Unresectable Hepatocellular Carcinoma. European Journal of Cancer, 47, 2117-2127. https://doi.org/10.1016/j.ejca.2011.05.007

[18] Li, X.L., Guo, W.X., Hong, X.D., Yang, L., Wang, K., Shi, J., et al. (2016) Efficacy of 
the Treatment of Transarterial Chemoembolization Combined with Radiotherapy for Hepatocellular Carcinoma with Portal Vein Tumor Thrombus: A Propensity Score Analysis. Hepatology Research, 46, 1088-1098. https://doi.org/10.1111/hepr.12657

[19] Lu, D.H., Fei, Z.L., Zhou, J.P., Hu Z.T. and Hao, W.S. (2015) A Comparison between Three-Dimensional Conformal Radiotherapy Combined with Interventional Treatment and Interventional Treatment Alone for Hepatocellular Carcinoma with Portal Vein Tumour Thrombosis. Journal of Medical Imaging and Radiation Oncology, 59, 109-114. https://doi.org/10.1111/1754-9485.12207

[20] Wang, K., Guo, W.X., Chen, M.S., Mao, Y.L., Sun, B.C., Shi, J., et al. (2016) Multimodality Treatment for Hepatocellular Carcinoma with Portal Vein Tumor Thrombus: A Large-Scale, Multicenter, Propensity Mathching Score Analysis. Medicine, 95, e3015. https://doi.org/10.1097/MD.0000000000003015

[21] Kang, J., Nie, Q., Du, R., Zhang, L., Zhang, J., Li, Q., et al. (2014) Stereotactic Body Radiotherapy Combined with Transarterial Chemoembolization for Hepatocellular Carcinoma with Portal Vein Tumor Thrombosis. Molecular and Clinical Oncology, 2, 43-50. https://doi.org/10.3892/mco.2013.196

[22] Liu, P.H., Lee, Y.H., Hsu, C.Y., Hsia, C.Y., Huang, Y.H., Chiou, Y.Y., et al. (2014) Surgical Resection Is Better than Transarterial Chemoembolization for Hepatocellular Carcinoma beyond Milan Criteria Independent of Performance Status. The Journal of Gastrointestinal Surgery, 18, 1623-1631. https://doi.org/10.1007/s11605-014-2546-9

[23] Zhao, Y.N., Zhang, Y.Q., Ye, J.Z., Liu, X., Yang, H.Z., Cong, F.Y., et al. (2016) Hepatic Resection versus Transarterial Chemoembolization for Patients with Barcelona Clinic Liver Cancer Intermediate Stage Child-Pugh A Hepatocellular Carcinoma. Experimental and Therapeutic Medicine, 12, 3813-3819.

https://doi.org/10.3892/etm.2016.3810

[24] Peng, Z.W., Guo, R.P., Zhang, Y.J., Lin, X.J., Chen, M.S. and Lau, W.Y. (2012) Hepatic Resection versus Transcatheter Arterial Chemoembolization for the Treatment of Hepatocellular Carcinoma with Portal Vein Tumor Thrombus. Cancer, 118, 4725-4736. https://doi.org/10.1002/cncr.26561

[25] Zheng, N., Wei, X., Zhang, D., Chai, W., Che, M., Wang, J., et al. (2016) Hepatic Resection or Transarterial Chemoembolization for Hepatocellular Carcinoma with Portal Vein Tumor Thrombus. Medicine, 95, e3959. https://doi.org/10.1097/MD.0000000000003959

[26] Lee, J.M., Jang, B.K., Lee, Y.J., Choi, W.Y., Choi, S.M., Chung, W.J., et al. (2016) Survival Outcomes of Hepatic Resection Compared with Transarterial Chemoembolization or Sorafenib for Hepatocellular Carcinoma with Portal Vein Tumor Thrombosis. Clinical and Molecular Hepatology, 22, 160-167. https://doi.org/10.3350/cmh.2016.22.1.160

[27] Choi, J.H., Chung, W.J., Bae, S.H., Song, D.S., Song, M.J., Kim, Y.S., et al. (2018) Randomized, Prospective, Comparative Study on the Effects and Safety of Sorafenib vs. Hepatic Arterial Infusion Chemotherapy in Patients with Advanced Hepatocellular Carcinoma with Portal Vein Tumor Thrombosis. Cancer Chemotherapy and Pharmacology, 82, 469-478. https://doi.org/10.1007/s00280-018-3638-0

[28] Song, D.S., Song, M.J., Bae, S.H., Chung, W.J., Jang, J.Y., Kim, Y.S., et al. (2015) A Comparative Study between Sorafenib and Hepatic Arterial Infusion Chemotherapy for Advanced Hepatocellular Carcinoma with Portal Vein Tumor Thrombosis. The Journal of Gastroenterology, 50, 445-454. 
https://doi.org/10.1007/s00535-014-0978-3

[29] Nakano, M., Niizeki, T., Nagamatsu, H., Tanaka, M., Kuromatsu, R., Satani, M., et al. (2017) Clinical Effects and Safety of Intra-Arterial Infusion Therapy of Cisplatin Suspension in Lipiodol Combined with 5-Fluorouracil versus Sorafenib, for Advanced Hepatocellular Carcinoma with Macroscopic Vascular Invasion without Extra-Hepatic Spread: A Prospective Cohort Study. Molecular and Clinical Oncology, 7, 1013-1020. https://doi.org/10.3892/mco.2017.1442

[30] Nagai, H., Mukozu, T., Ogino, Y.U., Matsui, D., Matsui, T., Wakui, N., et al. (2015) Sorafenib and Hepatic Arterial Infusion Chemotherapy for Advanced Hepatocellular Carcinoma with Portal Vein Tumor Thrombus. Anticancer Research, 35, 2269-2277.

[31] He, M., Li, Q., Zou, R., Shen, J., Fang, W., Tan, G., et al. (2019) Sorafenib Plus Hepatic Arterial Infusion of Oxaliplatin, Fluorouracil, and Leucovorin vs Sorafenib alone for Hepatocellular Carcinoma with Portal Vein Invasion: A Randomized Clinical Trial. JAMA Oncology, 5, 953-960.

https://doi.org/10.1001/jamaoncol.2019.0250

[32] Onishi, H., Nouso, K., Nakamura, S., Katsui, K., Wada, N., Morimoto, Y., et al. (2015) Efficacy of Hepatic Arterial Infusion Chemotherapy in Combination with Irradiation for Advanced Hepatocellular Carcinoma with Portal Vein Invasion. Hepatology International, 9, 105-112. https://doi.org/10.1007/s12072-014-9592-y

[33] Kodama, K., Kawaoka, T., Aikata, H., Uchikawa, S., Nishida, Y., Inagaki, Y., et al. (2018) Comparison of Outcome of Hepatic Arterial Infusion Chemotherapy Combined with Radiotherapy and Sorafenib for Advanced Hepatocellular Carcinoma Patients with Major Portal Vein Tumor Thrombosis. Oncology, 94, 215-222. https://doi.org/10.1159/000486483 